\title{
Femtosecond Laser Ablation in Burst Mode as a Function of Pulse Fluence and Intra-Burst Repetition Rate
}

\author{
Norman Hodgson, Hortense Allegre, Andrei Starodoumov, and Skip Bettencourt \\ Coherent, Inc. \\ 5100 Patrick Henry Drive \\ Santa Clara, CA 95054, USA \\ Corresponding author's e-mail address: norman.hodgson@coherent.com
}

\begin{abstract}
Ablation rates were measured in burst mode operation for various materials (metals, semiconductors, dielectrics) as a function of pulse fluence, intra-burst repetition rate $(60 \mathrm{MHz}, 180 \mathrm{MHz}$, $360 \mathrm{MHz}, 720 \mathrm{MHz}, 1.44 \mathrm{GHz}$ ) and the number of pulses per burst (1-30), using a $40 \mu \mathrm{J}, 1035 \mathrm{~nm}$ Yb:Fiber MOPA with 300 fs pulse duration and repetition rates between 100 and $250 \mathrm{kHz}$. Burst mode operation allows operation near the optimum fluence for laser ablation, thus maximizing the ablation rate. Depending on the material, number of pulses in the burst, intra-burst repetition rate and the ablation geometry, the ablation efficiency can be equal, lower or multiple times higher compared to non-burst operation. For metals and large area ablation, burst mode operation enables access to the maximum ablation rate by using more than 5 pulses per burst. Intra-burst repetition rates higher than $180 \mathrm{MHz}$, or using less than 5 pulses usually leads to a decrease in ablation efficiency due to shielding by the ejected material/plasma. For materials with low thermal conductivity, like glasses or plastics, and for semiconductors materials, interaction between burst pulses can substantially increase the maximum ablation rate over the 1-pulse case, leading to more efficient ablation as the number of burst pulses and the intra-burst frequencies are increased.
\end{abstract}

DOI: $10.2961 /$ jlmn.2020.03.2014

Keywords: ultrafast laser ablation, burst mode operation, femtosecond laser ablation, optimum fluence, heat affected zone

\section{Introduction}

Material processing with femtosecond pulses in the range of 300 to $900 \mathrm{fs}$ has gained in popularity due to the small heat affected zone (HAZ) and increased energy penetration depth resulting from the high pulse intensity. The vast majority of the industrial ultrafast laser processing applications are based on ablation, including cutting foils for flat panel displays, stents, drilling of fuel injector nozzles, wafer scribing and surface micro-structuring [1]. Surprisingly, the ablation rates for sub-ps pulses on materials that exhibit free electrons can be relatively high [2-6]. Compared to 20 ps long pulses, the ablation depths per pulse can be up to three times larger for femtosecond pulses, leading to maximum ablation rates that are only a factor of three lower as compared to Q-switched lasers with pulse durations of tens of ns. Ablation processes using fs and ps lasers therefore exhibit relatively high process speeds while generating a small heat affected zone. One challenge of ultrafast laser ablation is that the optimum fluences at which the highest ablation rates are achieved are relatively low. This is a result of the low ablation threshold fluences which for metals and a laser wavelength of around $1 \mu \mathrm{m}$ can be as low as $0.05 \mathrm{~J} / \mathrm{cm}^{2}$, and proportionally lower when shorter laser wavelengths down to the UV are used.

It is well known, that for pulsed laser ablation the maximum ablation rate is achieved at a pulse fluence is $\mathrm{e}^{2}$ times the ablation threshold fluence. For a constant laser power at the work piece, operation at the optimum fluence guarantees that the largest fraction for the incident power is used to ablate material (Fig.1). The ablation rate $\mathrm{C}$ in $\mathrm{mm}^{3}$ per min and per Watt of laser power as a function of the pulse fluence is given by $[5,6]$ :

$$
\mathrm{C}=\frac{30 \delta}{F}\left(\ln \left[\frac{F}{F_{t h}}\right]\right)^{2}
$$

where $F$ is the average pulse fluence in $J / \mathrm{mm}^{2}, F_{\text {th }}$ is the average threshold fluence and $\delta$ is the energy penetration depth in $\mathrm{mm}$. Please note that in this paper the average pulse fluence is always used, given as the ratio of pulse energy to the area of the Gaussian beam (spot diameter defined by the $1 / \mathrm{e}^{2}$ decrease in intensity).

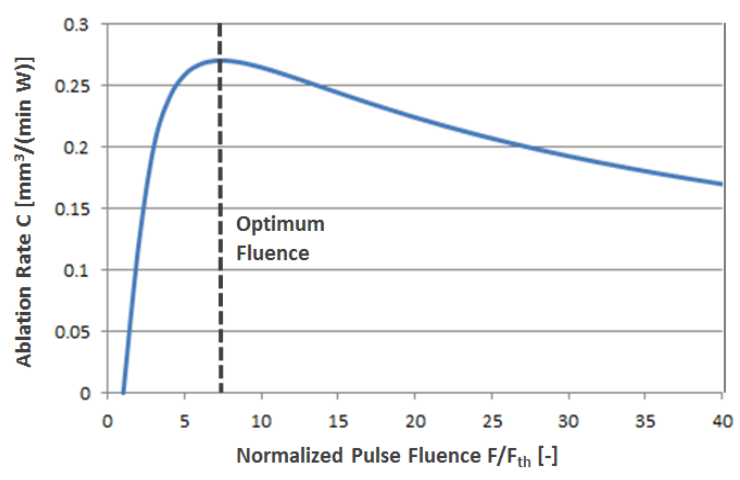

Fig. 1 Ablation rate as a function of pulse fluence for pulsed laser ablation using a Gaussian intensity profile and a ratio $\delta / F_{\text {th }}$ of $0.017 \mathrm{~mm}^{3} / \mathrm{J}$. 
Typical energy penetration depths for metals are between $20 \mathrm{~nm}$ and $40 \mathrm{~nm}$ for sub-ps pulses. The ablation rate exhibits a maximum at about 7.4 times the threshold fluence, and the ablation depth is twice the energy penetration depth at this point. Operation at the optimum fluence is important because the heat-affected zone is not only decreased but also becomes independent of the pulse duration for pulses shorter than $10 \mathrm{ps}$ [7].

Figure 2 shows measured maximum ablation rates versus the measured optimum pulse fluences for various materials at laser wavelengths of $1035 \mathrm{~nm}$ and $345 \mathrm{~nm}$ and pulse durations of $10 \mathrm{ps}$ and 400-500 fs, respectively [7]. It is apparent that average pulse fluences of around $1 \mathrm{~J} / \mathrm{cm}^{2}$ are required to maximize the ablation efficiency. For a typical laser output power of $100 \mathrm{~W}$ in the infrared, and a spot diameter of $25 \mu \mathrm{m}$, this means that the laser repetition rate has to be in the $10-20 \mathrm{MHz}$ range. However, due to limited scanner speeds, process repetition rates are typically around $500 \mathrm{kHz}$, leading to pulse fluences that can be forty times higher than the optimum.
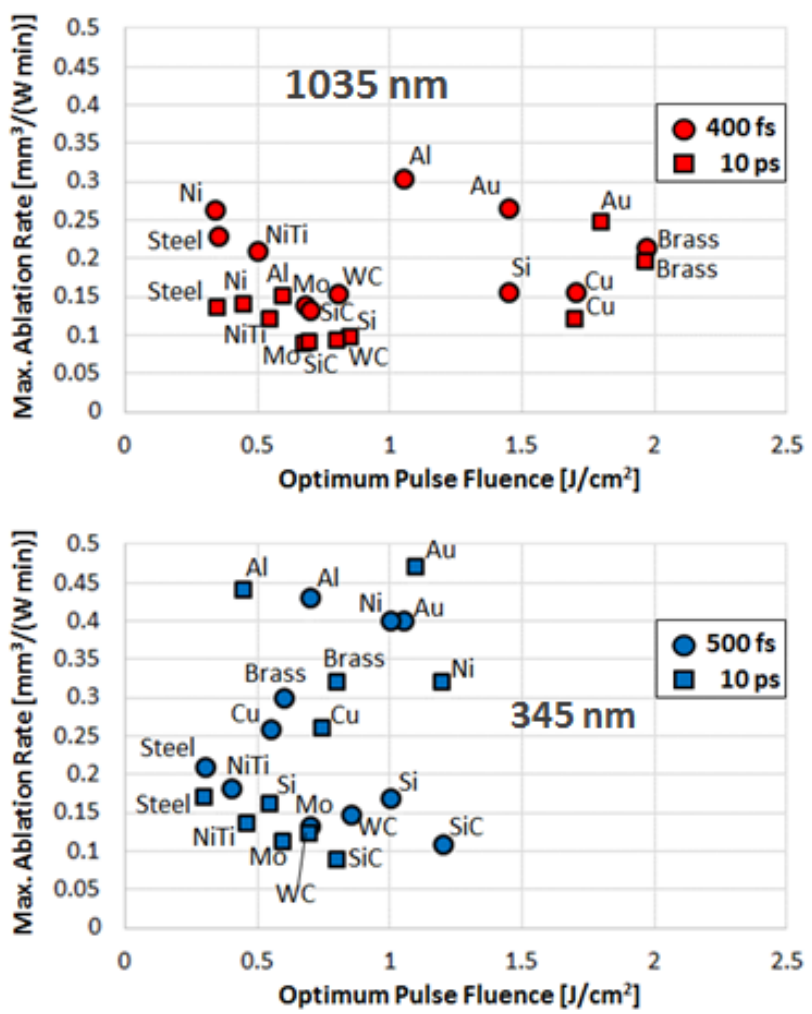

Fig. 2 Measured maximum ablation rates versus the optimum pulse fluences for various metals and semiconductors using pulse durations of 400-500 fs and 10 ps and laser wavelengths of $1035 \mathrm{~nm}$ and $345 \mathrm{~nm}$ [7]. Results are shown for large area ablation $(\sim 1.5 \times 0.5 \mathrm{~mm})$ with $60 \%$ spot overlap and about 400 ablated layers.

Operating at such a high pulse fluence leads to a reduction of the ablation rate by more than a factor of 3 . Using seeder burst mode operation with $\mathrm{N}$ pulses per burst will reduce the fluence of individual laser pulses by a factor of $\mathrm{N}$ thus moving the operating point towards the optimum fluence $[8,9]$. If high process repetition rates of tens of $\mathrm{MHz}$ are not feasible, seeder burst mode operation provides a viable method to increase the ablation rate, as operation near the optimum fluence will not only increase the material removal rate but will also reduce the heat affected zone [7-11].

For industrial laser processes, the relevant parameter is not the ablation rate, but rather the process speed, which is defined as the volume removal per minute. The process speed is obtained by multiplying the ablation rate (1) with the laser power. Figure 3 shows different ways to increase the process speed for ablation of copper. For a $60 \mathrm{~W}$ laser at $500 \mathrm{kHz}$ using a $25 \mu \mathrm{m}$ spot diameter, the initial pulse fluence of $24.5 \mathrm{~J} / \mathrm{cm}^{2}$ is about a factor of 10 above the optimum fluence and the process speed is around $5 \mathrm{~mm}^{3} / \mathrm{min}$. There are three ways to increase the process speed: Doubling the laser power at the same repetition (route 1), doubling both laser power and repetition rate (route 2), or using burst mode operation at $500 \mathrm{kHz}$ with 10 pulses per burst (route 3 ). Burst mode operation not only leads to the highest process speed of greater $10 \mathrm{~mm}^{3} / \mathrm{min}$, but it also requires only half the laser power as compared to the other two options.

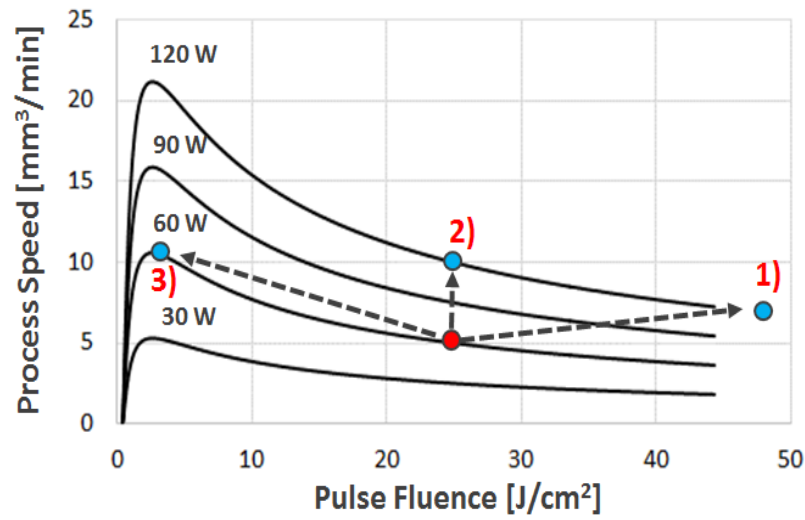

Fig. 3 Process speed for copper ablation as a function of the average pulse fluence. Curve parameter is the laser power. For a $60 \mathrm{~W}, 500 \mathrm{kHz}$ lasers with $25 \mu \mathrm{m}$ spot diameter, there are three options to increase the process speed. Option 3) represents burst mode operation with 10 pulses per burst.

The basic assumption here is that burst mode operation enables ablation rates that are equal to the ones that would be obtained when operating the laser at a high repetition of $10 \mathrm{~s}$ of $\mathrm{MHz}$ and at the optimum pulse fluence (Fig.4, route A). This means that we assume that there is no interaction between the intra-burst pulses via the work piece, which from a thermal perspective is true for most metals as the thermal relaxation time within the ablated spot is in the 1-2 ns range. In reality, however, the ablation rates may be lower due to plasma shielding of individual pulses (Fig.4, route B). For materials with low thermal conductivity, like glasses and polymers, the ablation rate can also be increased due to the thermal interaction between pulses (route $\mathrm{C}$ ).

Which of these three scenarios will be observed depends strongly on the material properties, the number of pulses per burst, the temporal spacing between pulses in the burst, and also on the geometry of the ablated area. Large ablation areas, for instance, will reduce plasma shielding effects, whereas cutting deep trenches does not allow the plasma plume to escape before the next pulse arrives. 


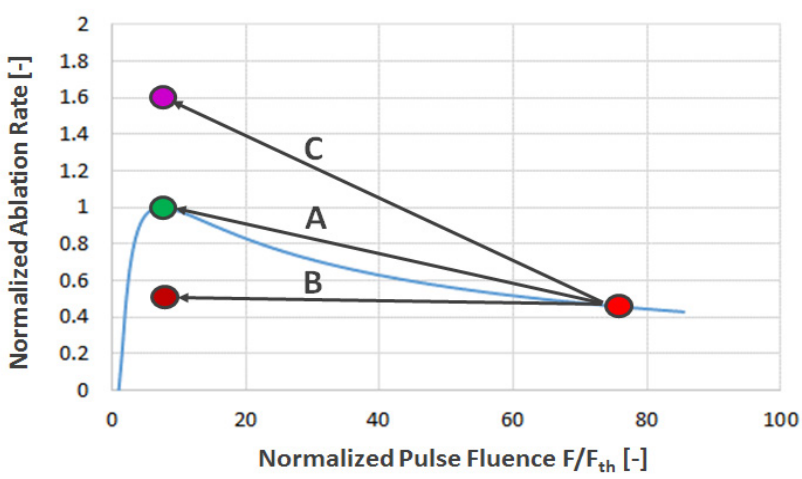

Fig. 4 Possible effects on the ablation rate $\mathrm{C}$ when switching on burst mode with ten pulses per burst at constant laser power. A) no interaction between pulses, B) plasma shielding between pulses, C) interaction between pulses leading to increased ablation depth per pulse.

We have measured the ablation rates for ten materials as a function of the pulse fluence using a $300 \mathrm{fs}$ infrared laser. Parameters that were varied in this study was the intraburst pulse spacing by using five seed laser repetition rate between $60 \mathrm{MHz}$ and $1.44 \mathrm{GHz}$, varying the number of pulses per burst from 1 pulse to up to 30 pulses. It is important to note that at a constant pulse fluence, the process speed will increase with the number of pulses in the burst. For copper at a pulse fluence of $2 \mathrm{~J} / \mathrm{cm}^{2}$, the process speed will be about $0.32 \mathrm{~mm}^{3} / \mathrm{min}$ for $2 \mathrm{~W}$ of laser power, whereas with a ten-pulse burst at this pulse fluence, the process speed will be ten times higher. In addition, we compared wide-area ablation with cutting a trench to understand the effect of the ablation geometry on the efficiency of burst mode ablation.

\section{Experimental Set-up}

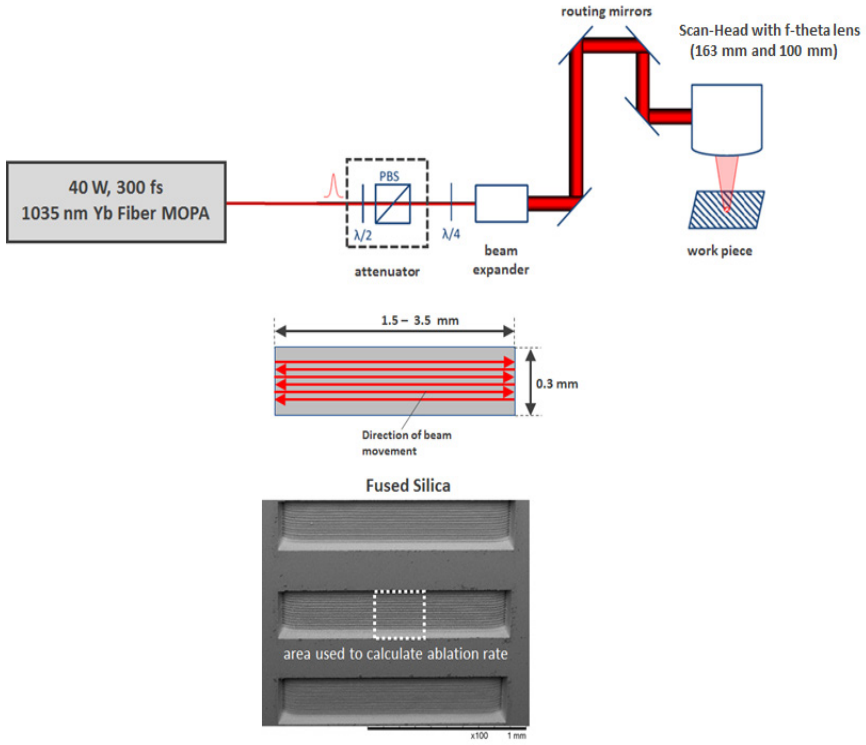

Fig. 5 Experimental set-up used to investigate laser ablation using burst mode operation of a $300 \mathrm{fs}$ Yb:fiber MOPA. The lower graphs show the large area ablation geometry. For all experiments a spot overlap of $60 \%$ was used in both directions.
We used a modelocked 300 fs Yb:fiber MOPA system providing a maximum pulse energy of $40 \mu \mathrm{J}$ and a maximum output power of $40 \mathrm{~W}$ at a wavelength of $1035 \mathrm{~nm}$ and a beam quality factor $\mathrm{M}^{2}$ of 1.1 . (Fig. 5). In order to implement different seed laser repetition rates, we used two different lengths $L$ of the gain fiber in the modelocked fiber oscillator. For fiber lengths of $1.72 \mathrm{~m}$ and $0.57 \mathrm{~m}$, the seed laser repetition rate was $60 \mathrm{MHz}$ and $180 \mathrm{MHz}$, respectively. Higher repetition rates were realized by using the 180 $\mathrm{MHz}$ seed laser and externally doubling the repetition rate using 50/50 beam splitters and fiber loops. Repeated external pulse interleaving resulted in repetition rates of 360 $\mathrm{MHz}, 720 \mathrm{MHz}$ and $1.44 \mathrm{GHz}$. The laser beam was attenuated using a rotatable half-waveplate and a polarization beam splitter, circularly polarized, and then focused onto the sample using a ScanLab scanhead with f-theta lenses of $163 \mathrm{~mm}$ and $100 \mathrm{~mm}$ focal lengths. The spot diameters on the sample were $23 \mu \mathrm{m}$ for the longer focal length and 13.5 $\mu \mathrm{m}$ for the $100 \mathrm{~mm}$ lens. The shorter focal length lens was used for seeder repetition rates of $180 \mathrm{MHz}$ and higher. Rectangular, $0.3 \mathrm{~mm}$ wide and $1.5-3.5 \mathrm{~mm}$ long cavities were ablated using a spot overlap of $60 \%$ in both directions. The typical depth of the cavities was around $100 \mu \mathrm{m}$. The central part of each a cavity was used to calculate the ablation rate by measuring the depth with a microscope. In addition, trenches were cut by scanning a line on the sample, again with $60 \%$ pulse overlap, and repeating the scan 150 times with a $1 \mathrm{~s}$ pause between individual scans.

\section{Experimental Results}

\subsection{Burst Mode Operation at $60 \mathrm{MHz}$}

Figure 6 shows the measured ablation rates for copper as a function of the pulse fluence for various number of pulses in the burst. Seeder repetition rate is $60 \mathrm{MHz}$, generating $16.7 \mathrm{~ns}$ temporal separation between pulses in the burst. The curves indicate that burst mode operation with 3, 5 and 10 pulses leads to a similar ablation rate as compared to using a single pulse.

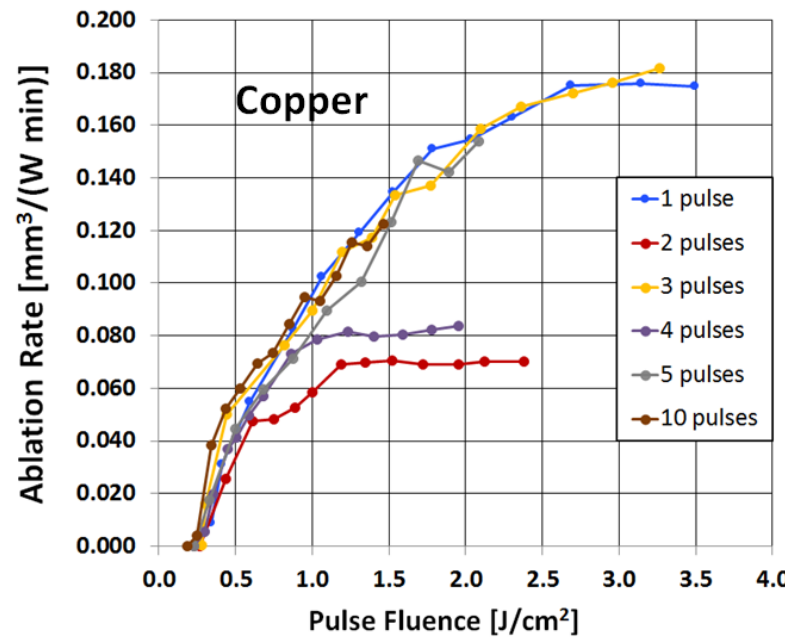

Fig. 6 Measured ablation rates of copper as a function of the pulse fluence and various numbers of pulses in the burst (at 60 $\mathrm{MHz}$ ). Process repetition rate was $250 \mathrm{kHz}$ for 1 to 4 pulses, and $200 \mathrm{kHz}$ for 5 and 10 pulses. Spot diameter: $23 \mu \mathrm{m}, 60 \%$ overlap. 
Our emphasis therefore is on the ablation rate rather than the process speed since a higher ablation rate will generally also lead to a smaller heat affected zone.

The lower ablation rates for 2 and 4 burst pulses in Fig. 6 are a result of plasma shielding, as a subsequent experiment varying temporal pulse separation revealed. This is shown in Fig. 7 where the measured ablation rates for 4pulse bursts with different temporal pulse sequences are compared. As can be seen, the shielding effect becomes less pronounced as the temporal separation of individual pulses in the burst is increased to more than $60 \mathrm{~ns}$. This shielding effect in for 2 and 4 burst pulses in copper has been extensively studied [12-14]. Time-resolved investigation of the shielding during copper ablation [14] showed that the lower ablation rate is a result of reflection at the plasma plume for even number of burst pulses.

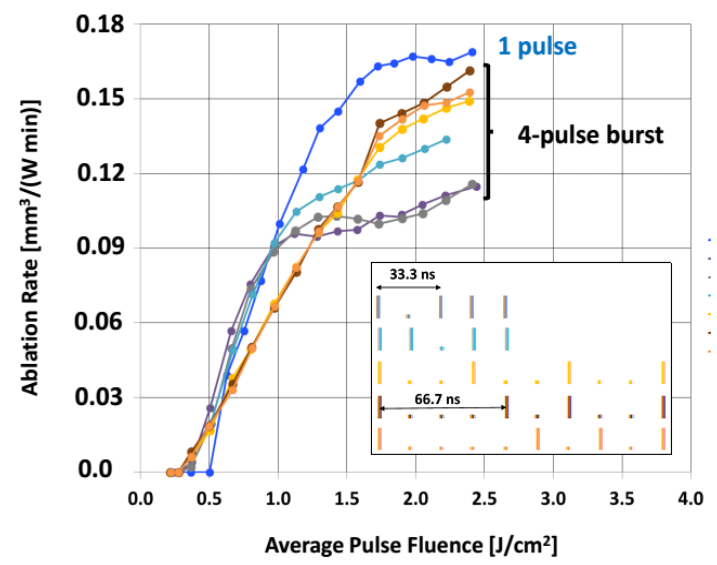

Fig. 7 Measured ablation rates of copper as a function of the pulse fluence for four-pulse bursts. The temporal sequence of the four pulses was varied according to the legend. The lower pink curve is the 4-pulse burst ablation rate with equal pulse spacing of $16.7 \mathrm{~ns}$. The process repetition rate was $250 \mathrm{kHz}$, spot diameter: $23 \mu \mathrm{m}, 60 \%$ spot overlap.

Measured ablation rates in burst mode operation for four different materials are shown in Fig. 8. Similar to copper, plasma shielding is evident for other materials, and particularly strong if 2 or 4 pulses in the burst are used. The shielding effect generally becomes less pronounced or negligible when more than 4 pulses are used. As a general rule, at least 5 pulses per burst should be used to avoid shielding effect when operating at seeder frequencies around $60 \mathrm{MHz}$. For silicon and fused silica, burst mode ablation leads to an increase of the ablation rate over the single-pulse case due to an interaction between the pulses (Fig. 9). For fused silica this can be explained by thermal interaction as the thermal relaxation time of around $10 \mathrm{~ns}$ is greater than the pulse separation. For silicon, with very high thermal conductivity, the interaction must be caused by a different, yet unknown, effect. Note that the ablation rate is enhanced by more than a factor of 2 when bursts with 5 or more pulses are used. This means that for the same laser power, and the same number of pulses per second, the ablation depth must be a twice as large in burst mode. In fact, measured ablation depths in fused silica were $43 \mu \mathrm{m}$ and $87 \mu \mathrm{m}$, when using $14.5 \mathrm{~W}$ of laser power with $10 \mathrm{ps}$ pulse duration and
$3.5 \mathrm{~J} / \mathrm{cm}^{2}$ pulse fluence, at $1 \mathrm{MHz}$ with 1 pulse and at $100 \mathrm{kHz}$ with a 10-pulse burst, respectively. In both cases, the process time was 7 seconds. A similar increase of the ablation depth was observed for silicon when switching from $1 \mathrm{MHz}$ single pulse operation to $100 \mathrm{kHz}, 10$ pulse burst mode with $6.5 \mathrm{~W}$ of laser power, $300 \mathrm{fs}$ pulse duration and a pulse fluence of $1.5 \mathrm{~J} / \mathrm{cm}^{2}$. These are two examples where burst mode operation actually increases both the process speed and the ablation efficiency as compared to the process speed that can be realized using single pulses at the same pulse fluence and the same average power (by increasing the repetition rate). This is in contrast to the majority of materials, including all metals, where burst mode is simply a mode of operation that optimizes the pulse fluence in order to achieve the same process speed as when using single pulse operation at high repetition rates.
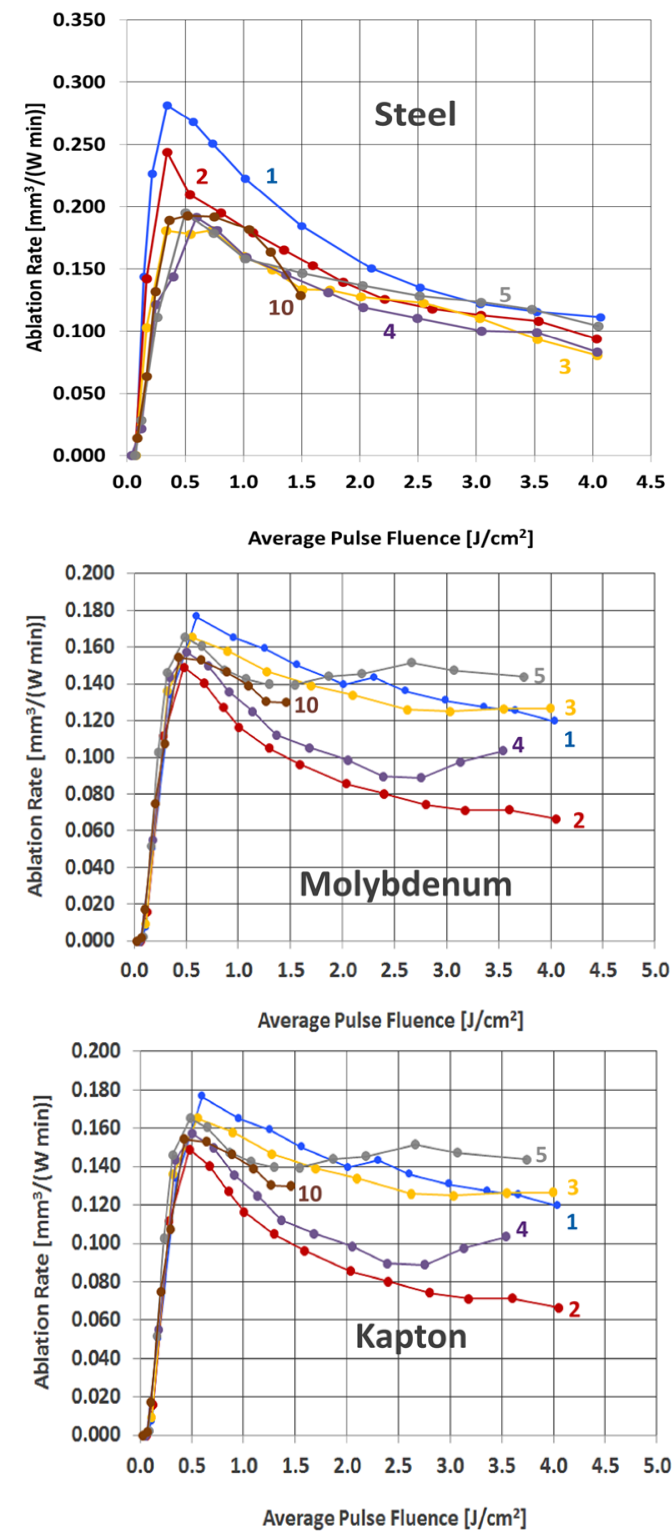

Fig. 8 Measured ablation rates of different materials as a function of the pulse fluence with varying number of pulses per burst. Curve parameter is the number of pulses per burst. Process repetition rate was $250 \mathrm{kHz}$ for 1 to 4 pulses, and $200 \mathrm{kHz}$ for 5 and 10 pulses. Spot diameter: $23 \mu \mathrm{m}, 60 \%$ spot overlap. 

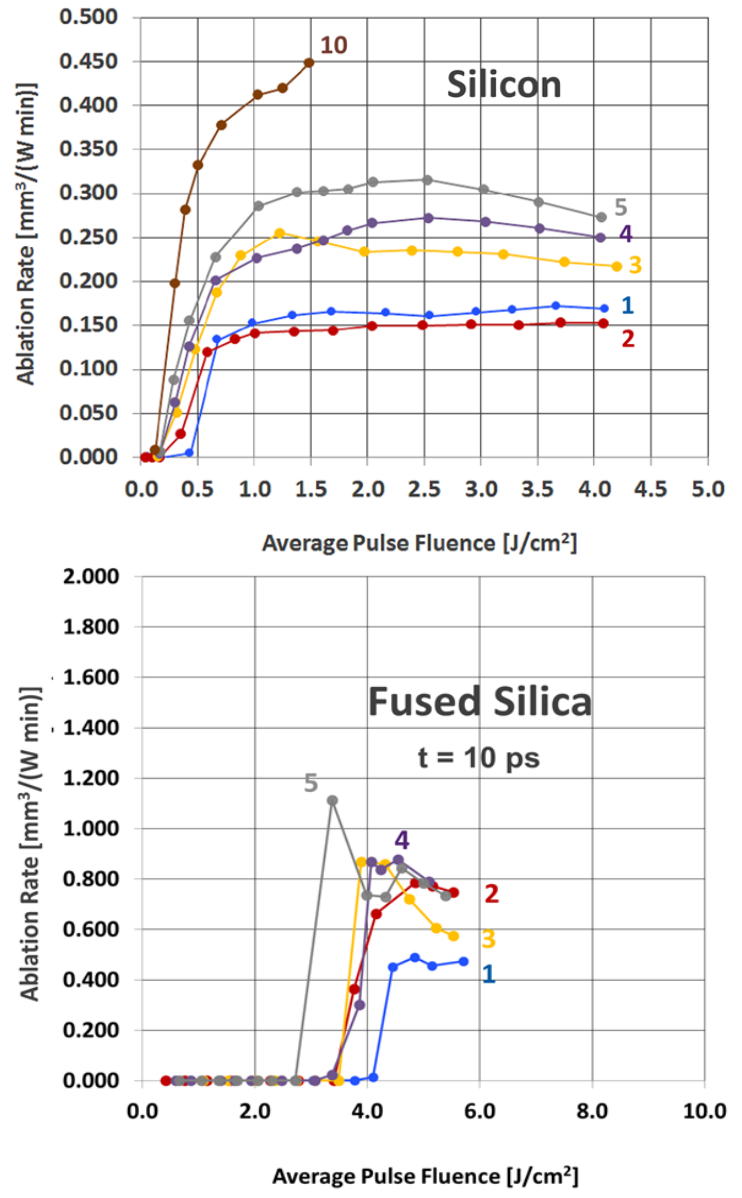

Fig, 9 measured ablation rates of silicon (300 fs pulses) and fused silica (10 ps pulses) as a function of the pulse fluence. Curve parameter is the number of pulses per burst. Process repetition rate was $250 \mathrm{kHz}$ for 1 to 4 pulses, and $200 \mathrm{kHz}$ for 5 and 10 pulses. Spot diameter: $23 \mu \mathrm{m}, 60 \%$ spot overlap.

Since plasma shielding is very detrimental for ablation, ablation geometries that do not allow fast dissipation of the plasma between pulses will not exhibit an increase in ablation rate when using burst mode. This is certainly true when cutting deep trenches into materials. Figure 10 shows the pattern used to generate trenches in steel, where line ablation with an increased number of parallel lines results in an increase of the ablated area. Each multi-line ablation pattern was repeated 150 times. When several lines are used, the ablated area is larger than the spot area thus allowing for lateral expansion of the plasma. The measured ablation depth as a function of the number of parallel lines is shown in Fig. 11. For a single ablated line, switching to a 10-pulse burst mode at the same laser power, decreases the ablation depth by $40 \%$, even though the pulse fluence is reduced from $3.7 \mathrm{~J} / \mathrm{cm}^{2}$ to $0.37 \mathrm{~J} / \mathrm{cm}^{2}$, and therefore close to the optimum fluence for ablation of steel. However, after opening up the area by using 5 parallel lines to a total width of about $70 \mu \mathrm{m}$, the penetration depth becomes twice as large compared to single pulse operation and stays constant when further increasing the ablated area. This clearly indicates that ablated areas next to the beam area need to be in place to allow the plasma to escape from the beam.

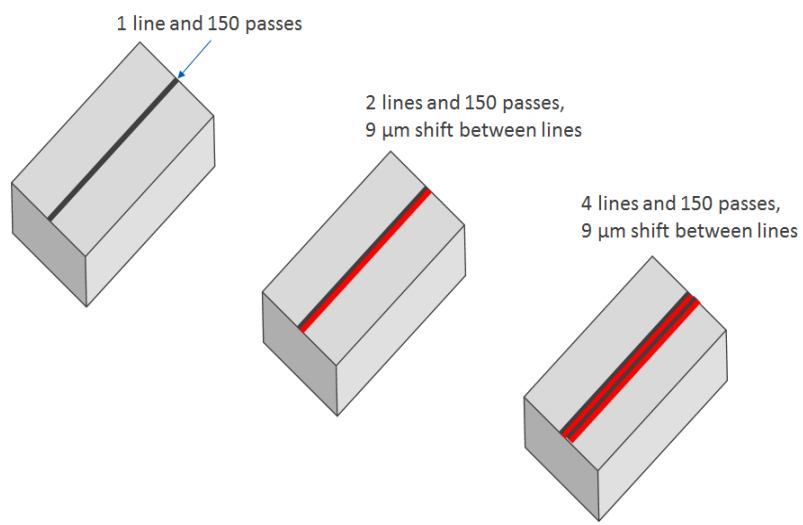

Fig. 10 Trench cutting using a line with $23 \mu \mathrm{m}$ spot diameter and $60 \%$ spot overlap, passing the same line 150 times (top left). Increasing the number of lines by shifting each new line by $9 \mu \mathrm{m}$ during each of the 150 repetitions.

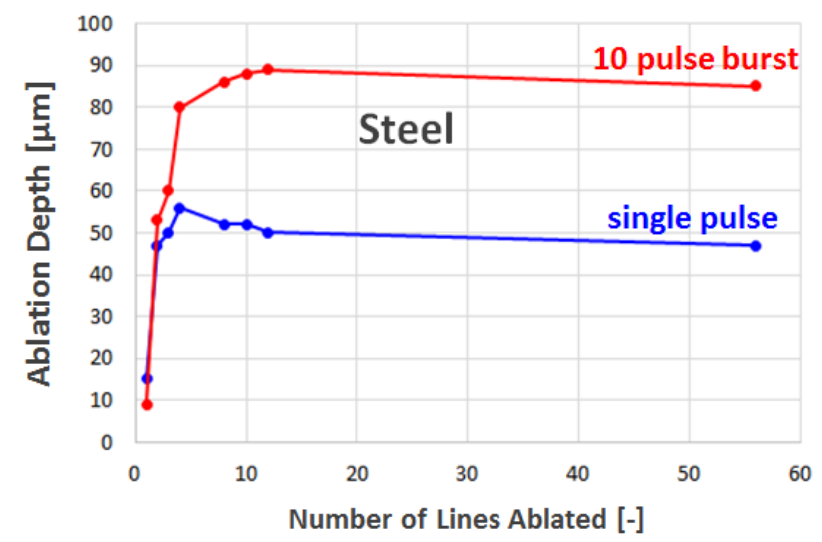

Fig. 11 Measured ablation depth in steel for line ablation using the pattern shown in Fig. 10 with 150 repetitions. Laser power: $3.1 \mathrm{~W}$, repetition rate of $200 \mathrm{kHz}, 23 \mu \mathrm{m}$ spot diameter, $60 \%$ spot overlap.

\subsection{Burst Mode Operation at High Seeder Repetition Rates}

In order to compare the ablation rates at higher seed repetition rates and for a larger number of pulses per burst (up to 30), the spot diameter was reduced to $13.5 \mu \mathrm{m}$ and the process repetition was kept constant at $200 \mathrm{kHz}$. This allowed operation at pulse fluences of up to $0.88 \mathrm{~J} / \mathrm{cm}^{2}$, even if 20 pulses per burst were used. At high seeder repetition rates of $360 \mathrm{MHz}, 720 \mathrm{MHz}$ and $1.44 \mathrm{GHz}$, the minimum number of pulses used were 10,20 and 30 , respectively. This is to account for the 9 ns rise and fall time of the pulse picker which generates a slope on the burst intensity envelope. The pulse fluences on the following graphs are the average of the pulse fluences in the burst, not including pulses that are below $20 \%$ of the maximum pulse intensity. Figures 12 and 13 show the measured ablation rates for metals as a function of the average fluence of the individual pulses for various intra-burst repetition rates and number of pulses per burst. As observed before, the general behavior is that burst mode does not increase the ablation rate, and shielding effects do become more pronounced as the temporal pulse spacing is decreased. 
This statement is certainly true for steel, molybdenum, gold, and titanium. However, for aluminum and copper, there are operational modes using bursts for which the ablation rates are increased compared to single pulse operation. For copper, an intra-burst frequency of $180 \mathrm{MHz}$ and 10 pulses per burst clearly enhances the ablation efficiency, indicating that for a pulse spacing of around $5 \mathrm{~ns}$, an interaction between pulses must take place on the sample, leading to an increase in the ablated volume per pulse. A less pronounced increase in ablation rate is observed for aluminum at 360 $\mathrm{MHz}$ and $720 \mathrm{MHz}$. Note that the maximum of the ablation rate generally moves to lower pulse fluences. This is a result of incubation due to the increased total number of pulses being incident on each location of the sample.

For semiconductors, and for dielectrics with low thermal conductivity, burst mode operation typically leads to an enhancement of the ablation rate due to interaction of the intra-burst pulses on the workpiece. For these materials, burst mode operation is clearly superior to ablation using single pulses at the optimum pulse fluence. Figure 14 shows this for kapton, silicon, and silicon carbide. For silicon, the ablation rate is increased by almost a factor of 4 , which means that at the same laser power, burst mode operation with 15 pulses in the burst and $\sim 3$ ns pulse spacing will lead to a process speed that is 4 times higher as compared to an optimized single pulse process. For semiconductors, and for dielectrics with low thermal conductivity, burst mode operation typically leads to an enhancement of the ablation rate.

Translating the ablation rates of silicon in Fig. 14 into a practical example, let us assume $20 \mathrm{~W}$ of laser power at a repetition rate of $1 \mathrm{MHz}$ and a spot diameter of $25 \mu \mathrm{m}$, generating a pulse fluence of $4.07 \mathrm{~J} / \mathrm{cm}^{2}$. According to Fig. 14 , the ablation rate of about $0.18 \mathrm{~mm}^{3} /(\mathrm{W} \mathrm{min})$ will result in a process speed of $3.6 \mathrm{~mm}^{3} / \mathrm{min}$. Switching on burst mode with 15 pulses at $360 \mathrm{MHz}$, will reduce the pulse fluence to $0.27 \mathrm{~J} / \mathrm{cm}^{2}$, resulting in an increase of the process speed to $12 \mathrm{~mm}^{3} / \mathrm{min}$. Note that silicon carbide, and silicon are the only materials where the ablation rate is increased using $\mathrm{GHz}$ intra-burst repetition rates. For all the other materials we investigated, 1 ns or sub-ns spacing between burst pulses will lead to a substantial decrease in the ablation rate. For the vast majority of materials, the best performance in burst mode operation seems to be attained if the temporal separation between pulses is larger than $5 \mathrm{~ns}$.

It is interesting to compare the measured ablation rates for copper and silicon in Figs. 12 and 14 with those published by Kerse et al. in 2016 [15]. By dividing the ablation rates by a factor of 60 , we get the removed volume in $\mathrm{mm}^{3}$ per Joule of incident laser energy and can compare with the results presented in Fig. 2c and 2d of [15]. Kerse at al. used only one burst to drill a hole with a beam spot diameter of 24 microns. The number of pulses per location is therefore much lower than in our large area ablation geometry with hundreds of two-dimensional scans. Despite the obvious difference in ablation geometry and number of pulses used per location, the ablation rates for silicon are similar, as the comparison of the maximum ablation rates and optimum pulse fluences shown in Fig. 15 indicates. For, copper, however, our ablation rates are much lower when using burst mode at a high intra--burst repetition rate, indicating that we observed much more pronounced plasma shielding.
We also did not observe any enhancement of the ablation rate as compared to the single-pulse case for copper. The measured ablation rates for copper in single-pulse mode are similar to those reported by Neuenschwander et al. [6], and in burst mode operation, we measured similar ablation rates for $\mathrm{Cu}, \mathrm{Al}$, and steel as Jaeggi et al. [12].
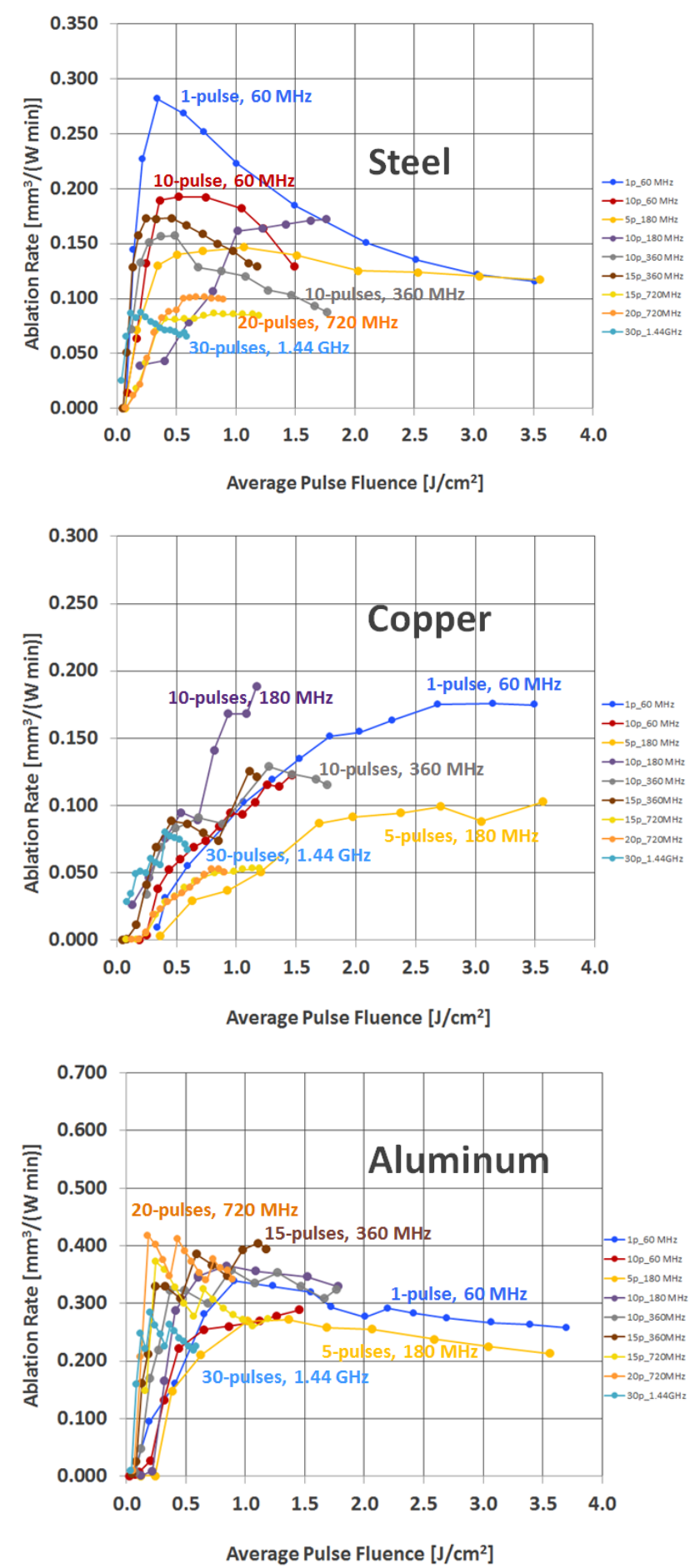

Fig. 12 Measured ablation rates for steel, copper, and aluminum as a function of the individual pulse fluence using different numbers of pulses per burst and different intra-burst repetition rates. Process repetition rate was $200 \mathrm{kHz}$. Spot diameter: 13.5 $\mu \mathrm{m}, 60 \%$ spot overlap. 

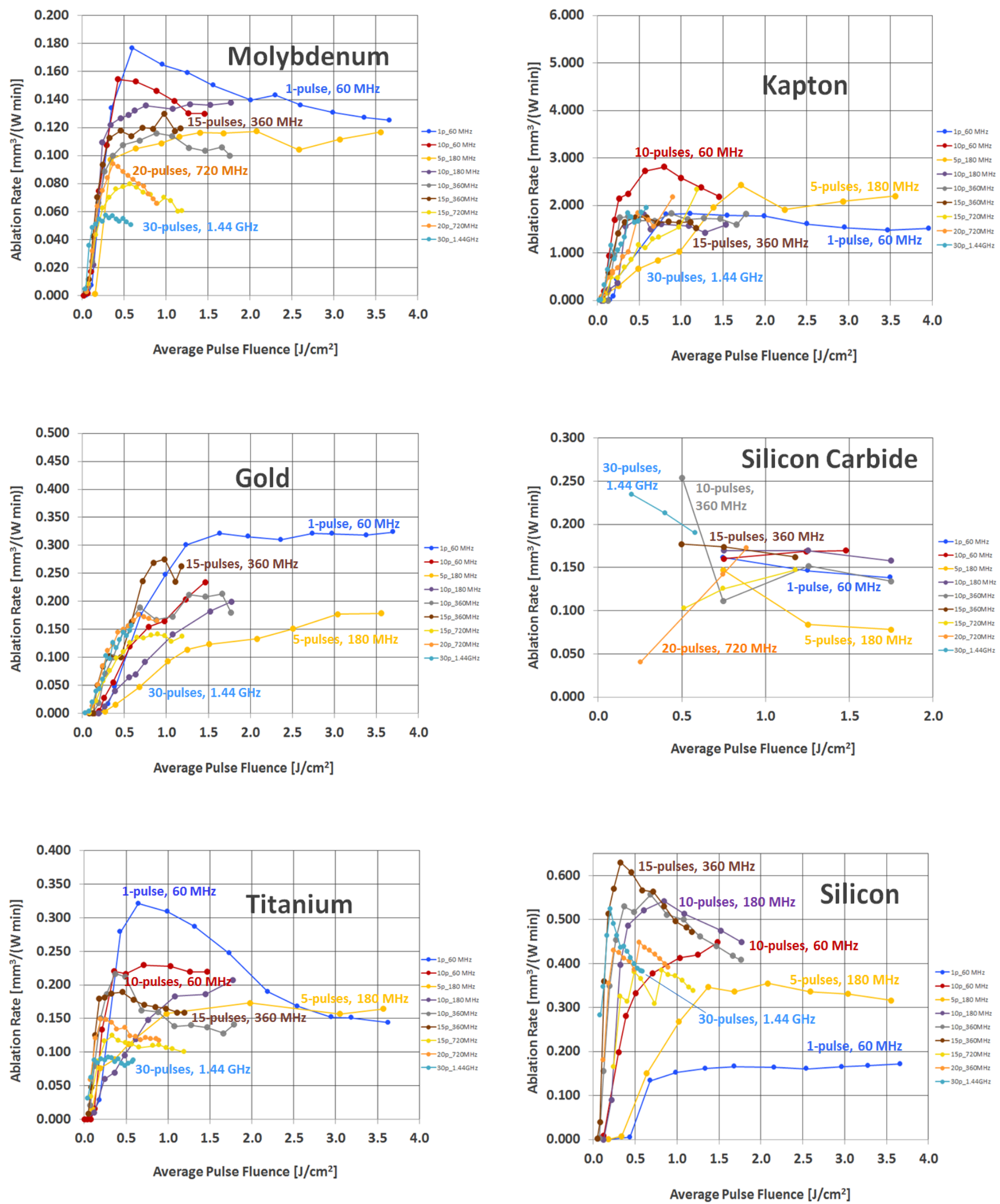

Fig. 13 Measured ablation rates for molybdenum, gold, and titanium as a function of the individual pulse fluence using different numbers of pulses per burst and different intra-burst repetition rates. Process repetition rate was $200 \mathrm{kHz}$. Spot diameter: $13.5 \mu \mathrm{m}, 60 \%$ spot overlap.

Fig. 14 Measured ablation rates for kapton, silicon carbide and silicon as a function of the pulse fluence using different numbers of pulses per burst and different intra-burst repetition rates. Process repetition rate was $200 \mathrm{kHz}$. Spot diameter: $13.5 \mu \mathrm{m}$, $60 \%$ spot overlap. 


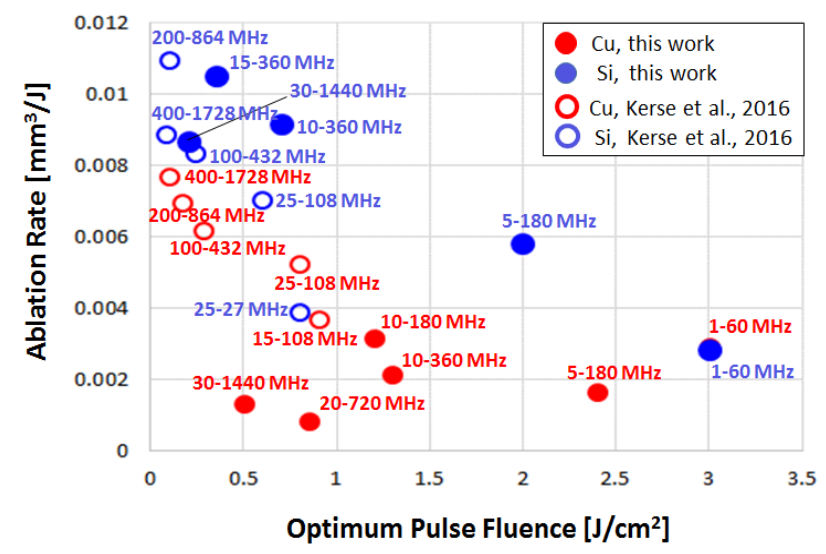

Fig. 15 Measured maximum ablation rates as a function of the optimum fluence for copper and silicon as shown in Figs 12 and 14, and as reported by Kerse et al. [15], for various number of burst pulses and intra-burst repetition rates. For each data point, the number of pulses in the burst and the intra-burst repetition rate are indicated.

Figure 16 summarizes the experimental results of the parametric burst mode operation study. The graph shows the measured maximum ablation rates for the ten materials studied under various operational conditions of the burstmode. For metals, burst mode operation does generally not increase the ablation rate, meaning that the highest process speed can be attained by using single pulse mode at the optimum pulse fluence. This, however, usually requires a process repetition rate in the $10-20 \mathrm{MHz}$ range.

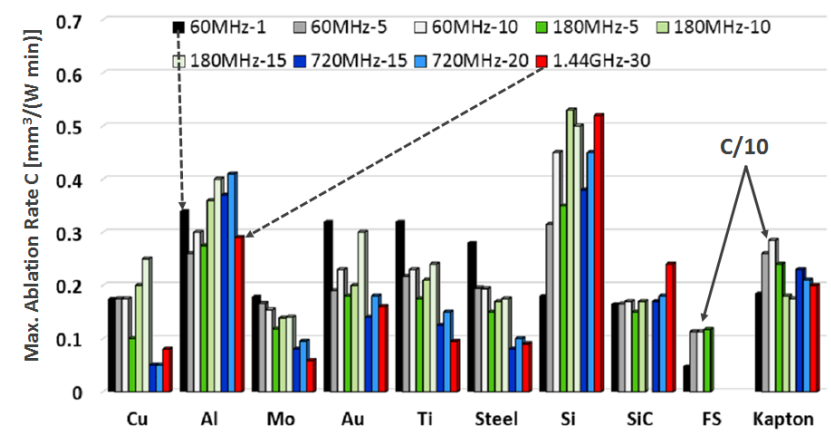

Fig. 16 Measured maximum ablation rates for ten materials for various intra-burst repetition rates and number of pulses per burst, as indicated in the legend.

If the process repetition rate has to remain in the sub-MHz range, burst mode operation will allow an increase in process speed by reducing the pulse fluence to get closer to the optimum fluence. For semiconductors and dielectrics, burst mode operation will, in addition, lead to an enhancement of the ablation rate compared to single pulse operation. For silicon, this enhancement can be as high as a factor of 4 . For metals and dielectrics, intra-burst repetition rates of greater than about $200 \mathrm{MHz}$ do not provide an advantage in regards to the maximally attainable ablation rates. $\mathrm{GHz}$ burst mode seems to only result in an increase in ablation efficiency for semiconductor materials and dielectrics with low thermal conductivity. However, even if the ablation rates in burst mode do not get close or exceed the maximum ablation rates in single pulse operation, burst mode operation will generally increase the process speed for focusing conditions where the pulse fluence is much higher than the optimum fluence for ablation of the material.

\section{Summary and Conclusion}

We have investigated the ablation efficiency in burst mode operation for ten different materials as a function of the pulse fluence and the intra-burst repetition rate using a 40 W Yb:fiber MOPA with $300 \mathrm{fs}$ pulse duration at $1035 \mathrm{~nm}$. For typical laser powers of up to $100 \mathrm{~W}$ and process repetition rates in range of around $200-1,000 \mathrm{kHz}$, burst mode operation allows ablation near the optimum pulse fluence, which for metals and semiconductor materials is around 1 $\mathrm{J} / \mathrm{cm}^{2}$. Burst mode operation thus enables maximization of the ablation rate and minimization of the heat affected zone for laser processing set-ups where high repetition rate operation in the range of $10-20 \mathrm{MHz}$ is not feasible. Shielding effects impose a lower limit on the number of pulses per burst. In general, having more than 5 pulses leads to similar maximum ablation rates as for single pulse operation. Using 2, 3 or 4 pulses in the burst may result in considerably lower ablation rates. For semiconductors and dielectrics (silicon, silicon carbide, fused silica, kapton), burst mode operation resulted in ablation rates that even exceeded the maximum rates for single pulse operation by up to a factor of 4 . This indicates that individual pulses interact with one another via the ablation process on the sample, leading to a larger ablated volume per pulse. Operation in burst mode requires that the plasma can escape out of the beam path between pulses. Large area ablation therefore is an ideal process to take advantage of burst mode operation, while trench cutting and hole-drilling requires that the ablated area is larger than the beam area to make burst mode work. With the exception of silicon, silicon carbide and fused silica, burst mode operation at intra-burst repetition rates greater than about $200 \mathrm{MHz}$ does not generate higher ablation rates.

In summary, the results presented indicate that burst mode operation is a viable option for ultrafast laser micromachining to maximize the process speed and minimize the heat affected zone.

\section{References}

[1] N. Hodgson, M. Laha, T. S. Lee, A. Steinkopff, and S. Heming, Industrial Femtosecond Lasers and Material Processing, in: Industrial Laser Solutions, PennWell Publishing, (2019) January 22.

[2] C. Momma, B.N. Chichkov, S. Nolte, F. von Alvens leben, A. Tuennermann, H. Welling, and B. Wellegehausen, Opt. Commun., 129, (1996) 134.

[3] S. Nolte, C. Momma, H. Jacobs, A. Tuennermann, B.N. Chichkov, B. Wellegehausen, and H. Welling, J. Opt. Soc. Am. B, 14, (1997) 2716.

[4] K.-H. Leitz, B. Redlingshoefer, Y. Reg, A. Otto, and M. Schmidt, Physics Procedia, 12, (2011) 230.

[5] B. Jaeggi, B. Neuenschwander, M. Schmid, M. Muralt, J. Zuercher, and U. Hunziker, Physics Procedia, 12, (2011) 164. 
[6] B. Neuenschwander, B. Jaeggi, M. Schmid, V. Rouffiange, and P.-E. Martin, Proc. SPIE, Vol. 8243, (2012) 824307.

[7] N. Hodgson, S. Heming, A. Steinkopff, H. Haloui, and T.S. Lee, Lasers in Manufacturing (LIM 2019), (2019) paper Tu_A_31-6.

[8] G. Raciukaitis, M. Brikas, P. Gecys, B. Voisiat, M. Gedvilas, J. Laser Micro/Nanoengin., 4, (2009) 186.

[9] R. Knappe, H. Haloui, A. Seifert, and A. Nebel, Proc. SPIE, Vol. 7585, (2010) 7585-16.

[10]B. Neuenschwander, B. Jaeggi, and M. Schmid, Physics Procedia, 41, (2013) 794.

[11] A. Zemaitis, M. Gaidys, M. Brikas, P. Gecys, G. Raciukaitis, and M. Gedvilas, Sci. Rep., 8, (2018) 173766.

[12]B. Jaeggi, S. Remind, Y. Zhang, T. Kramer, and B. Neuenschwander, J. Laser Micro/Nanoengin., 12, (2017) 258.

[13]D.J. Foerster, S. Fass, S. Groeninger, F. Bauer, A. Michalowski, and E.R. Weber, T. Graf, Appl. Surf. Sci., 440, (2018) 926.

[14]B. Bornschlegel and J. Finger, J. Laser Micro/Nanoengin., vol. 4, (2019) 88.

[15]C. Kerse, H. Kalaycioglu, P. Elahi, B. Cetin, D. K. Kesim, Ö. Akcaalan, S. Yavas, M. D. Asik, B. Öktem, H. Hoogland, R. Holzwarth, and F. Ö. Ilday, Nature, $537,(2016) 84$

(Received: July 17, 2020, Accepted: November 13, 2020) 